\title{
La clase interpretadora
}

\author{
Hernán G. Bouvier
}

CONICET-UNC

1. Voy a plantear tan solo tres cuestiones a "El escepticismo ante las reglas replanteado" de Riccardo Guastini. En primer lugar haré una breve consideración con miras a proponer una clave de lectura sobre la "teoría" de Guastini. Incidentalmente plantearé una duda. Pero lo que me interesa es realzar una forma de leer la propuesta general del texto bajo discusión. En segundo lugar, quiero detenerme en las consideraciones explicativas sobre la multiplicidad de interpretaciones y los condicionantes del pensamiento de quienes aplican las normas (puntos 1.5 y 2.1 de Guastini). En tercer lugar, voy a formular una pregunta genuina al autor. Entiendo por pregunta genuina aquella que se hace sin contenido retórico, frente a cuyo objeto no se tiene una posición tomada y para la cual no se avizora una respuesta obvia. Dicho de manera frontal, nada de trucos.

2. Guastini distingue entre tres tipos diferentes de ambigüedades que rodean al vocablo "interpretación". La manera de disolver estas ambigüedades es distinguir. 
La primera ambigüedad es disuelta con la distinción entre "in abstracto" o dirigida a textos e "in concreto" dirigida a hechos. La segunda ambigüedad se resuelve distinguiendo entre tres tipos de interpretación: la cognitiva, la decisoria y la creativa.

La cognitiva releva o identifica los diferentes usos o significados atribuidos a textos jurídicos según clases de reglas (semánticosintácticas, técnicas interpretativas, tesis dogmáticas, etc.); la decisoria elige entre alguno de estos significados; la creativa atribuye al texto un significado no comprendido en los ya relevados de manera descriptiva. La decisoria y creativa suponen una operación política (entrecomillada por Guastini), y si es llevada a cabo por un juez resulta auténtica (nuevamente entrecomillada). Con esta distinción Guastini acaba con la segunda ambigüedad que rodea al vocablo interpretación.

En el apartado sobre la tercera ambigüedad referente al vocablo interpretación se ocupa de las diferentes operaciones que llevan a cabo juristas y jueces. Distingue expresamente entre interpretación propiamente dicha y construcción. La interpretación propiamente dicha consiste en "la atribución de significados normativos (en alguno de los sentidos y modos que he señalado más arriba)". Quizás al utilizar "atribución" Guastini excluye del concepto de interpretación propiamente dicha a la cognitiva. Esto no es claro. Pero en todo caso nos deja el siguiente resultado: interpretación propiamente dicha es, al menos, la decisoria y la creativa.

Este primer tipo de "operación" (o primer sentido en que puede usarse interpretación aquí) debería ser distinguido del segundo tipo de operación que consiste en la construcción. La distinción no me resulta clara del todo ${ }^{1}$.

1 No es del todo claro cómo las operaciones constructivas (hipótesis contrafácticas sobre la intención del legislador, ratio legis, construcción de normas no expresas, etc) son independientes de las operaciones de interpretación propiamente dicha. Tomemos el caso de las normas noexpresas. O bien la norma no expresa es una consecuencia lógica extensional de algún significado atribuido al texto de acuerdo a alguna técnica o clase de reglas (semánticas, pragmáticas, convencionales de variado tipo) y puede ser relevado de manera cognitiva, en cuyo caso estamos en la interpretación descriptiva o cognitiva. $\mathrm{O}$ bien se ha elegido entre algunas de las 
Lo que parece claro es lo siguiente: que hay un marco interpretativo (la disyunción de interpretaciones posibles de un mismo texto) que divide aguas. Interpretaciones posibles aquí no es cualquier conjetura sobre el

interpretaciones posibles relevables cognitivamente ( $\mathrm{T}$ expresa N1) con lo cual estamos en la decisoria; o bien se ha dado un paso más allá del marco, Rahmen, cornice, ingresando en una interpretación creativa. No se ve muy bien cómo la construcción no implica al menos alguno de los sentidos de interpretación propiamente dicha. El ejemplo sobre la forma republicana del Estado (art. 139 Constitución Italiana) que da Guastini al hablar de construcción y normas no expresas confirma la duda aquí expuesta. Según Guastini, la mayoría de los constitucionalistas asume por definición que (para resumir) si es republicano entonces es democrático. Aquí claramente Guastini ha relevado en sentido descriptivo una de las interpretaciones que se hace de ese vocablo de acuerdo a un conjunto de reglas o creencias compartidas (la de los constitucionalistas). Si una Corte o un jurista "elige" ese sentido dejando de lado otro sentido posible de "republicano" ha operado una interpretación decisoria. Si nadie ha dicho jamás en la comunidad analizada que republicano equivale a democrático (lo cual Guastini no puede decir porque ha concedido que hay gente que lo hace) entonces hay interpretación creativa. ¿Cuál es la diferencia entre construcción e interpretación propiamente dicha? Se me escapa. No veo cómo la construcción de una norma no-expresa no implique alguno de los sentidos de interpretación propiamente dicha. Quizás las "operaciones de construcción" son una condición de posibilidad de la operación decisoria o la creativa o quizás una relación "proceso-producto". No lo sé. La cuestión se agrava un poco, aunque no es grave ni tan importante. Al hablar de interpretación decisoria vs. creativa, se pone como ejemplo de interpretación creativa aquella que equipara "Italia es una República democrática" a "Italia es una monarquía Absoluta" (pto 2.4). Con un poco de imaginación se ve que el ejemplo de interpretación creativa es traducible al ejemplo "república igual a democracia" utilizado por Guastini para hablar de construcción. Si "democracia igual monarquía absoluta" como ejemplo de interpretación creativa, es también un caso de construcción, se sigue que no hay límite a la distinción propiamente dicha vs. constructiva. Pero los límites de "propiamente dicha" están confusos, además, porque Guastini a renglón seguido de este ejemplo de "democracia igual a monarquía absoluta" sostiene que "cada atribución de significado que caiga fuera del marco de los significados admisibles no constituye propiamente una interpretación, sino más bien la creación de una norma nueva". En definitiva, el problema es cuál es la relación entre los predicados "interpretación propiamente dicha", "creación" y "construcción". 
texto, según parece. Se trata de algunas de las interpretaciones establecidas de manera más o menos persistente o bien por reglas semánticas, pragmáticas, o por tesis dogmáticas o de algún tipo de clase de sujetos a delimitar (lingüistas, juristas, etc.). Si se toma una de las interpretaciones posibles, se decide; si se salta fuera de este marco, se crea (o construye según entiendo). Es aquí donde toman relevancia las consideraciones sobre indeterminación.

Según Guastini, a la indeterminación jurídica contribuyen no solo problemas estructurales del lenguaje (u objetivos como los llama Guastini con comillas agregadas) sino también la multiplicidad de métodos interpretativos, la dogmática jurídica y el sentimiento de justicia de los intérpretes. Los juristas y jueces disponen de toda una complejidad para atribuir a un texto diferentes significados y de hecho lo hacen. No se puede discutir demasiado esta tesis de hecho, según creo. Sin embargo, queda mucho espacio para discutir los términos de la descripción.

En efecto, para otra forma de ver el derecho, si los jueces o los abogados crean derecho, se mueven en un ámbito en el que o bien no son competentes o bien esa conducta está prohibida (o ambas). Para dejar de lado esta cuestión podría responderse alegando la contingencia de ésta o aquella norma de competencia o de éste o aquel Código Penal. No creo que esta vía nos lleve muy lejos, y casi seguro nos devuelve al punto de partida. Si el carácter de "contingente" de un fenómeno excluye la validez de la teoría que lo analiza o describe, eso también invalida la teoría que acepta que el derecho es un fenómeno históricamente situado. Y creo que un positivista jurídico como Guastini acepta que el derecho es un fenómeno históricamente situado, contingente. Podría haber sido de otra manera. Podríamos tener un mundo sin derecho.

Pero resumiendo, la indeterminación del derecho es local: viene dada por algunos problemas del lenguaje y por el tipo de práctica (y sujetos que la integran), los cuales han adquirido la capacidad de interpretar e interpretar e interpretar. En todo caso, como el propio Guastini remarca, no queda aquí huella de escepticismo global o frente a todo el lenguaje y las reglas. 
¿En qué consiste entonces la teoría de Guastini? En una suma elegante y creativa de las siguientes tesis: a. existe un marco, cornice o Rahmen interpretativo cuyo límite puede venir dado por un cierto acuerdo o convergencia; b. el derecho está indeterminado por algunas particularidades del lenguaje y (sobre todo) por cómo juegan a ese juego sus jugadores; c. la práctica jurídica está conformada por los juristas y jueces que podrían muy bien ser denominados la clase interpretadora. Esta clave de lectura del realismo genovés surge de readaptar un rótulo utilizado por Donoso Cortés para criticar a los liberales (y que agrada a Carl Schmitt).

La afirmación según la cual los juristas y jueces forman parte de una clase interpretadora importa un sentido descriptivo (a diferencia de la idea de Donoso Cortés quien rotula a los liberales como "la clase discutidora" en claro sentido peyorativo). Creo que hablar de clase interpretadora capta la idea general que tiene Guastini sobre el derecho.

3. Contar con una clase interpretadora que apela a ciertas herramientas (métodos interpretativos, dogmática jurídica) o está embebida de preferencias ético políticas genera variedad de interpretaciones. La variedad de interpretaciones genera indeterminación. Soslayar este punto, sostiene Guastini, resulta crítico y criticable.

Las teorías criticadas por Guastini desatienden dentro de este contexto dos aspectos importantes: en primer lugar que los defectos estructurales u "objetivos" del lenguaje son solo una parte de lo que explica la indeterminación jurídica; en segundo lugar, que hay que ocuparse de más cosas que los problemas de indeterminación e interpretación judicial.

El gesto teórico de detenerse tan solo en la interpretación judicial relega a segundo plano cuestiones centrales. Entre ellas: la interpretación de los juristas; su participación en la clase interpretadora; y su capacidad de moldear la forma de pensamiento de los jueces (punto 2.1).

La idea del todo plausible de que la formación específica de los jueces condiciona sus pensamientos e interpretaciones constituye 
claramente una explicación ambiental del fenómeno de la pluralidad de interpretaciones ${ }^{2}$. Considero que habría que hacer un agregado.

El ambiente de formación no solo tiene capacidad de instalar ideas en varios sujetos. En muchos casos es autoreferencial. Uno de los sentidos en el que resulta autoreferencial es el siguiente: en muchos lugares los jueces hacen dogmática y/o dan clases y los dogmáticos pueden aspirar a jueces. Es más, la condición de "respetable académico" funciona actualmente como una condición contribuyente del acceso al cargo $^{3}$. Círculos semejantes de conectividad comunicativa y afectiva pueden encontrarse con los abogados-catedráticos y su actividad ante los tribunales. No los voy a explorar. Cabría agregar, además, que existen ideologías que defienden y han defendido como valiosa la influencia de los juristas. La influencia de hecho que han tenido los juristas en los procesos de producción de normas ha sido utilizada en sentido valorativo positivo, como una salvaguarda técnica de cierta tradición del pueblo frente a la volatilidad y ligereza de otros tipos de representación democrática parlamentaria ${ }^{4}$.

Esta capacidad de condicionamiento de los juristas y jueces por juristas y jueces, sin embargo, es una parte del complejo fenómeno que moldea la forma mentis. Guastini no dice nada sobre este aspecto más que lo ya indicado. Pero hablamos de condicionamientos -esto es, de explicaciones- y aquí faltan muchas cosas.

2 “..., los jueces, antes de asumir su cargo, han frecuentado la facultad de derecho y esto implica que el pensamiento de los juristas -las teorías dogmáticas y los métodos interpretativos- condiciona las ideas que los jueces poseen acerca del derecho en general, de los conceptos jurídicos y de la interpretación (de hecho, condiciona la propia forma mentis de los jueces)".

3 O cuanto más no sea para defender como imparcial o racional el nombramiento en cuestión.

${ }^{4}$ Sobre la retórica que rodeaba en Alemania al rol del jurista como técnico representante del pueblo y protector frente a la voluntad el parlamentarismo, véase Baratta, Alessandro. "Le fonti del diritto de il diritto giurisprudenziale" en Materiali per una storia della cultura giuridica vol XX, n.1, giugno 1990. pp. 189-210. La escuela genovesa, claro está, no apoya ideológicamente esta incidencia de los juristas y dogmáticos sobre la producción de normas. Sólo la describe. 
No caben dudas que los juristas que forman parte de la dogmática y la academia (profesores, comentadores, doctrinarios de variado tipo) han influido e influyen en la forma de pensar de quien es o será juez. Sin embargo, es importante notar que el tipo de influencia que tenía el dogmático o académico se ha debilitado sensiblemente. Esto se debe a lo que podríamos llamar el paso del espíritu comunitario al societario. Es decir, el paso de grupos sociales (más o menos pequeños), unidos por lazos de familia, cercanía y afecto personal, a grupos de tipo societario con la impronta de anonimidad e impersonalidad de las relaciones. No se puede soslayar este punto.

La influencia que tuvo la doctrina y la dogmática sobre la jurisprudencia (y que todavía conserva en parte) se explicaba por cierta cercanía entre jueces y comentaristas, propia de grupos en donde prima un tipo de relación afectiva (y "noble") de familia de juristas que se conocen entre sí, y que refuerzan sus lazos de pertenencia a partir de una práctica de respeto y pleitesía mutuo. El paso del espíritu comunitario o afectivo al societario o anónimo (Tönnies) genera que comience a modificarse o extinguirse ese tipo de influencia que la doctrina ejerció sobre la jurisprudencia. Tomemos por caso el crecimiento exponencial de las universidades. En Italia, según parece, dentro del top ten de Atenei più richiesti se encuentra Roma 1 La Sapienza y la Università degli Studi di Milano (primer y cuarto puesto respectivamente). La Sapienza cuenta con 147.000 inscriptos y Milano contaba hace 10 años (período 2001-2002) con 60.294. Génova, si entiendo bien, para el período 2009-2010 contaba con algo así como 35.000 estudiantes. Niente male ${ }^{5}$.

Es probable que esto se traduzca también en un número alto de estudiantes de Derecho. No creo que haga falta mostrar que, salvo vaivenes, el número de estudiantes de Derecho ha ido in crescendo en los últimos 50 años. La discusión sobre cómo controlar el número de inscriptos en abogacía y de abogados (la discusión sobre numerus clausus vs. matrícula libre) es una consecuencia y síntoma de que existe un crecimiento exponencial que cambia la relación de formación y la relación entre juristas y jueces.

${ }^{5}$ Puede verse www.universitaitaliane.it 
Pero hay más. ¿Qué hay acerca de lo que podríamos llamar "la profecía de la burocracia de Weber"? Esto es, el crecimiento exponencial del aparato administrativo y la complejidad de competencias de infinitos tipos de funcionarios. El nombre "Buro-cracia", más que un rótulo, resume en forma de descripción definida un fenómeno que debe tomarse en serio al momento de hablar de los condicionamientos de la decisión y/o interpretación. El siglo XX ha multiplicado exponencialmente el número de agentes, funcionarios y empleados que administran el poder político y jurídico. El número de agentes capaces de incidir en el proceso de creación de normas, asignación de significados a textos, etc., ha explotado. El énfasis en el par "doctrinario-juez" resulta ya poco luego del ochocientos ${ }^{6}$.

El common mind jurídico está conformado por más ideas que las que pertenecen al dueto parroquial-universitario de "doctrinario-juez".

En universidades masivas y administraciones hipertrofiadas, la relación entre jurista-alumno-juez está severamente mediada, distanciada, y consecuentemente la influencia que tienen unos sobre otros.

Hasta aquí, entonces, la observación referente a la incidencia del paso de la comunidad a la sociedad (del afecto al anonimato) y la referente a la multiplicación de funcionarios a través de la expansión de la burocracia.

Tampoco se encuentran en Guastini referencias a otros fenómenos obvios que condicionan el pensamiento de los jueces y que hacen que

${ }^{6}$ Además, la racionalidad burocrática tiende a expulsar (aunque nunca elimina completamente) rasgos propios de grupos comunitarios. El afecto, la familiaridad, la tradición y cualquier otro componente de cercanía y parcialidad tienden a ser excluidos por la "imparcialidad" técnica la cual, a su vez, maximiza algún tipo de "igualdad". Por último, la estructura racionalburocrática resiste, disuelve o expulsa las decisiones por mero gusto, capricho personal o cualquier forma de subjetividad libre y azarosa. Es decir, limita a los componentes personales que la integran, atrapándolos dentro de sus propias reglas. Esto quiere decir que "el sentimiento de justicia del intérprete, sus preferencias" tiende a ser licuado o embretado en un aparato burocrático. Weber, M. Schriften zur Soziologie, Stuttgart, Reclam, 1995. Edición de M. Sukale. pp.238-256. 
opten por esta o aquélla interpretación. En primer lugar, no se hace mención a las acciones estratégicas de los jueces (para ascender, no ser expulsado, no quedar en la picota mediática o precisamente lo opuesto, lograr visibilidad mediática). Este tipo de acciones estratégicas que dan lugar a diversas y novedosas interpretaciones, están condicionadas no por lo que dicen juristas o dogmáticos sino por otro tipo de horizontes, fines o fenómenos. De tal modo que los condicionamientos aquí no son teórico-universitarios, sino diferentes.

Otro tanto vale para nuevos fenómenos. Por ejemplo, la incidencia de organismos multi-pluri-nausea-level con capacidad de dejar en claro a una cierta organización judicial lo que debería hacer (cómo interpretar) para cumplir con una serie de compromisos internacionales ${ }^{7}$.

No me parece que todas estas cuestiones indicadas estén ya implicadas por las fuentes principales de indeterminación jurídica a las que alude Guastini. Y tampoco me parece que estén implicadas por las consideraciones que Guastini hace acerca de lo que condiciona la interpretación judicial y la forma mentis de los jueces.

4. Por último, Guastini sostiene que la teoría del derecho debe dedicarse a describir y a desplegar actividades de tipo cognitivo.

${ }^{7}$ Especialmente agudo es este fenómeno cuando un organismo financiero internacional condiciona un crédito a ciertas garantías de reforma, eficacia o transparencia del Poder Judicial. Para cumplir con ese tipo de lineamientos se favorece desde (y en) los tribunales superiores la aplicación de la ley de un cierto modo, lo cual condiciona, nuevamente (y de manera novedosa) la forma mentis. Eficiencia, sustentabilidad y accountabilities de variado tipo son conceptos que han ingresado a la práctica judicial desde hace poco (rompiendo su hermética cerrazón intramuros propia de los tribunales del pasado que no debían dar cuentas en términos de eficiencia). La clásica expresión según la cual "los tiempos de la justicia son lentos" (léase "los tiempos de los jueces son lentos"), que en su momento tenía la carga valorativa de indicar que las decisiones de los jueces son y deben ser lentas y meditadas, ha adquirido ahora un matiz peyorativo. Pero claro que para acelerar ciertas causas hay que cambiar, entre miles de cosas, la interpretación sobre qué se entiende por "plazo razonable del proceso". Y lo que parece condicionar este cambio de interpretación no es lo que los jueces aprendieron en la universidad. 
Pregunto ¿por qué? Si la respuesta es que si algo no es descriptivo o cognitivo deja de ser una teoría del derecho, mi pregunta concede la definición y se transforma en ¿por qué deberíamos hacer teoría del derecho? ¿Es útil, conveniente, valioso, razonable, a cosa serve? ¿Por qué y para qué hacemos lo que hacemos? Mejor dicho, la pregunta es personal, ¿Riccardo, por qué habría que hacer teoría del derecho? Tocca ate. 